IOSR Journal of Pharmacy

ISSN: 2250-3013, www.iosrphr.org

||| Volume 2 Issue 5 ||| Sep-Oct. 2012 \||| PP.08-11

\title{
Use of naloxone for the management of opioid overdose
}

\author{
Tinh Nguyen, Pharm.D. Candidate ${ }^{1}$, Elizabeth Englin, Pharm.D. Candidate ${ }^{1}$, \\ Whitney Palecek, Pharm.D. Candidate ${ }^{1}$, Eric Wombwell, Pharm.D., BCPS ${ }^{1,2}$ \\ ${ }^{1}$ University of Missouri - Kansas City, School of Pharmacy,Kansas City, Missouri USA; ${ }^{2}$ Centerpoint \\ Medical Center, Independence, Missouri USA
}

\begin{abstract}
Opioid overdose is a potentially life-threatening condition that requires immediate medical attention, often landing affected individuals into the emergency department. The longstanding treatment for opioid overdose has been naloxone, a pure opioid antagonist that reverses all signs of opioid intoxication. While effective as a reversal agent, naloxone has a half-life which is shorter than all opioids. Once the effect of naloxone wears off, patients may experience recurrence of opioid intoxication and the consequences can be fatal. The objective of this article is to review the available literature that addresses routes of administration and doses of naloxone as well as the necessary duration of observation when treating patients who present with opioid overdose. The preferred route of administration remains intravenous with intramuscular and intranasal considered alternatives. Recommended dosing is dependent upon history and the opioid being reversed; however, doses range from $0.05-2$ milligrams. A literature search revealed a relatively small number of studies related to observation periods following naloxone administration and subsequent recurrence of opioid toxicity. The available literature supports incorporation of a two-hour observation period following administration of naloxone for short-acting opioids and a longer four to six hour observation period for reversal of long-acting opioids.
\end{abstract}

Keywords —naloxone, narcotic antagonist, opioid intoxication, opioids, overdose

\section{INTRODUCTION}

Opioids exert their analgesic effect by binding to opioid receptors that exist throughout the central and peripheral nervous system. The end result is inhibition of transmission of nociceptive information [1-3]. Opioids also cause euphoria, specifically by an agonistic effect on the $\mu$-opioid receptors located in the mesocorticolimbic system [4]. Because of this property, opioid analgesics are often misused. Opioid overdose is becoming a more prevalent issue in our society, with mortality rates more than triple from 5.3\% per year before 1990 to $18.1 \%$ per year from 1990 to 2002 [5]. Between 1997 and 2007, prescriptions for opioid analgesics increased by $700 \%$ in the United States [6]. In 2008, more than 36,000 deaths were reported from drug overdose, with prescription opioid analgesics, cocaine, and heroin being the most common [7]. Opioids are also frequently used in hospitals and are commonly associated with medication errors [8]. Results from a 2007 national reporting system showed that more than half $(53 \%)$ of opioid errors resulted in overdoses, and many cases (22\%) required intervention with an opioid antagonist [9].

\section{SIGNS AND SYMPTOMS}

Patients with opioid intoxication will exhibit classic signs of depressed mental status (ranging from drowsiness to coma), decreased respiratory rate, decreased bowel sounds, and constricted pupils. Hypotension, bradycardia, convulsions, and hypothermia may also be present, especially in polysubstance overdose [1,10-12]. The cardinal sign of opioid overdose is a respiratory rate less than 12 breaths per minute in a patient who is not in physiologic sleep $[1,10]$.

\section{PHARMACOKINETICS OF OPIOID OVERDOSE}

Management of opioid overdose can be problematic due to the differences in pharmacokinetic properties of each agent. This can be compounded by the accumulation of pills into masses, called bezoars, after large ingestions that produce unpredictable rates of absorption. In addition, opioid overdose leads to saturation of the hepatic enzymatic system, shifting the elimination kinetics from first-order to zero-order, thus prolonging toxicity [10]. 


\section{MANAGEMENT}

Airway support and restoration of breathing are essential before proceeding with pharmacological intervention [14,15]. Once the airway is secured, administration of an opioid antagonist is the next step to reverse opioid toxicity. Naloxone, a short-acting competitive $\mu$-opioid receptor antagonist, is the antidote for opioid overdose. The onset of action of naloxone depends on its route of administration. The preferred route is intravenous (IV) because it provides the most rapid effect, usually within 2 minutes [16], although anecdotal response is seen within 30-60 seconds. Alternative routes such as intramuscular (IM) or subcutaneous (SC) have an effect within 3-5 minutes [17], and the intranasal (IN) route may take up to 13 minutes [15]. A few studies have been conducted on the efficacy of these alternative routes. Sporer et al demonstrated that patients had similar response rates to $2 \mathrm{mg}$ naloxone given IM as compared with IV [18]. Wanger et al compared 0.8mg given SC and $0.4 \mathrm{mg}$ given IV. The results showed no difference in time interval from arrival at patient's side until respiratory rate rose above 10 breaths per minute [19]. However, the authors may have incorrectly concluded that there was no difference when one may have existed as the study did not meet power. Both studies pointed out that the IM and SC routes provide erratic absorption and delayed elimination [18,19]. However, these routes are acceptable alternatives when the IV route is not readily accessible $[11,14,16]$. A 2009 study by Kerr et al demonstrated that time to adequate response was comparable between IN and IM naloxone [20]. In a recent retrospective study, Weber et al concluded that nebulized naloxone is safe and effective based on an $80 \%$ response rate [21]. The oral route is not used due to extensive first-pass metabolism, producing negligible bioavailability [10]. The duration of action for naloxone is 30-60 minutes, and the terminal half-life is $30-90$ minutes. It is primarily metabolized by the liver and excreted as inactive metabolites in the urine $[13,14$, $16,19]$.

The initial dose of naloxone ranges from $0.05 \mathrm{mg}$ to $2 \mathrm{mg}$ and is dependent upon the severity of respiratory depression and the patient's history of opioid dependence [11]. A lower starting dose is typically used in opioid-dependent patients to avoid precipitation of withdrawal [11,16,22], while higher doses may be required to restore respiration in bradypneic or apneic patients [11,22]. A practical starting dose of $0.4 \mathrm{mg}$ IV should reverse most opioid-induced respiratory depression $[1,2,11,13,14,16,22]$. Patients with cardiopulmonary arrest require the highest dose of $2 \mathrm{mg}$ to effectively reverse opioid toxicity, regardless of patient's drug use history [11,23]. Dose titration should occur every 2-3 minutes until normal respiration returns (12 or more breaths per minute) $[1,2,11,13,14,16,22]$. There is no maximum dose for naloxone in the absence of opioid withdrawal, however, an alternative diagnosis should be considered after $10 \mathrm{mg}$ of naloxone $[1,2,11,14,16,22]$.

\section{POST-NALOXONE ADMINISTRATION}

Naloxone has a shorter half-life than all opioids, therefore it is logical to monitor patients for recurrence of opioid toxicity $[11,24]$. However, there is no consensus on a specific time frame to observe patients in the emergency department (ED) before discharge.

In a prospective, observational study, Christenson et al examined the safety of early discharge decisions and the accuracy of outcome prediction by physicians 1 hour after administration of naloxone for patients with presumed opioid overdose. Patients were included if they received naloxone in the prehospital or ED setting. Exclusion criteria were death in the ED within 1 hour after receiving naloxone, left the ED against medical advice, or refused to consent to follow-up. 573 patients were eligible for this study, the mean age was 35.7 years and the majority were males (82\%). At 1 hour assessment, ED physicians indicated that 282 patients $(49.2 \%)$ were safe for discharge based on the following "prediction rule": 1) ability to mobilize; 2) normal $\mathrm{O}_{2}$ saturation $(>92 \%)$; 3) normal respiratory rate (>10, and $<20$ breaths per minute); 4) normal temperature $\left(>35^{\circ} \mathrm{C}\right.$ and $<37.5^{\circ} \mathrm{C}$ ), 5) normal heart rate ( $>50$ and $<100$ beats $\left./ \mathrm{min}\right)$; and 6) Glasgow Coma Scale $\geq 15$. Only $6(2.1 \%)$ of these patients suffered an adverse event after discharge. Overall, 278 patients (48.5\%) stayed less than 2 hours in the hospital. This duration reflects extra time that patients were held to address social, psychiatric, and other issues after they were considered safe for discharge. 130 patients (22.7\%) stayed between 2 and 4 hours, and $165(28.8 \%)$ stayed more than 4 hours. The authors concluded that the 1 hour post-naloxone administration "prediction rule" safely assessed patients with presumed opioid overdose for early discharge. However, validation of this rule is required prior to clinical use [25].

In a retrospective cohort study, Boyd et al looked at records of 145 eligible patients with presumed heroin overdose treated by emergency medical services (EMS) personnel. The mean age was 26 years and the majority were males $(82.8 \%)$. ED evaluation did not occur in $84 / 145$ patients, with 71 of those 84 (85\%) having received prehospital naloxone. All 71 patients showed no signs of hypoventilation following naloxone administration and none reported life-threatening events during a 12-hour follow-up. Only 61/145 patients (42\%) were transferred to the ED after prehospital treatment. Of these, 29 were discharged, 8 left against medical advice, 23 were admitted to the hospital, and 1 was transported elsewhere. There was no mention of the time between ED admission and discharge. The authors concluded that allowing patients to leave after 
prehospital naloxone treatment is safe and a 1 hour observation period after naloxone administration in the ED is adequate for presumed heroin overdose [26].

Watson et al conducted a retrospective case-control study of naloxone-treated patients with opioid toxicity over a period of 8 years. Their objective was to determine the recurrence rates of opioid toxicity after an initial response to naloxone. Recurrence of opioid toxicity was defined as lethargy or somnolence during ED stay. The study included 84 patients with a mean age of 33 and the majority (58\%) were males. Other baseline characteristics were similar between groups. Patients were observed in the ED for a mean duration of 6 hours. The results revealed that 42 patients (50\%) responded to naloxone. Of these, $13(31 \%)$ had recurrence of opioid toxicity. After an initial response to naloxone, recurrence of opioid toxicity was seen between 3 and 120 minutes. Patients who took hydromorphone, meperidine, morphine immediate-release, or pentazocine-all short-acting opioids - had no recurrence of toxicity. The authors concluded that frequency of opioid toxicity recurrence is approximately $20-45 \%$ after an initial response to naloxone and recurrence of opioid toxicity is more frequent with long-acting opioids [24].

Since long-acting opioids are of particular concern for recurrence of opioid toxicity, patients with known overdose of long-acting opioids (e.g. methadone) or sustained release products (e.g. OxyContin) should be admitted to the hospital for extended observation. When reversing the effects of longer-acting opioids, a continuous infusion of naloxone may be necessary to prevent recurrent toxicity [14,22,27]. In one case report, a 17 year old patient with methadone overdose received a total of $3.2 \mathrm{mg}$ of naloxone IV boluses over a 13-hour period but continued to revert back to unconsciousness with periods of apnea. He was then started on a naloxone drip of $0.8 \mathrm{mg} /$ hour overnight. By the next morning, the patient was alert, cooperative, and functional [28]. Watson et al demonstrated that patients who took long-acting opioids were significantly more likely to experience recurrence of toxicity than those who took short-acting opioids. Hospitalization and continuous naloxone infusion were more prevalent in patients who experienced recurrence of opioid toxicity [24]. In a pharmacokinetic study, Goldfrank et al provided a dosing nomogram for those who require prolonged opioid antagonism with naloxone. The authors suggested using an hourly continuous infusion rate that is $66 \%$ of the initial administered bolus dose to prevent recurring opioid toxicity [27]. Overall, the duration of observation following naloxone administration to treat opioid overdose should be based on the half-life of the ingested opioid, the patient's vital signs, oxygen saturation, mental status, and the physician's clinical judgment $[11,14,22,25]$.

\section{CONCLUSION}

Opioid overdose causes significant morbidity and can be a life-threatening condition. Naloxone is an effective antidote that reverses opioid toxicity when given quickly, at adequate doses. Due to its short duration of action compared to opioids, multiple doses may be required to fully reverse toxicity. In cases of short-acting opioid overdoses, patients may be discharged once respiration and mental status return to normal and an observation period of 2 hours has been conducted after naloxone administration. For long-acting or sustainedrelease opioid overdoses, hospital admission is required and a continuous infusion of naloxone may be warranted. The observation period in these cases may extend to 4-6 hours after naloxone infusion and until the patient is awake and alert with no signs of respiratory depression.

\section{REFERENCES}

[1] A. Fareed, S. Stout, J. Casarella, S. Vayalapalli, J. Cox, and K. Drexler, Illicit opioid intoxication: diagnosis and treatment, Subst Abuse, 5, 2011, 17-25.

[2] L.S. Nelson, Opioids, in N. E. Flomenbaum (Ed.), Goldfrank's toxicologic emergencies, 38 (New York: McGraw Hill, 2006) 590-613.

[3] A.M. Trescot, S. Datta, M. Lee, and H. Hansen, Opioid pharmacology, Pain Physician, 11, 2008, S133-S153.

[4] G.F. Koob, and M. Le Moal, Drug addiction, dysregulation of reward, and allostasis, Neuropsychopharmacology, 24(2), 2001, 97-129.

[5] L.J. Paulozzi, D.S. Budnitz, and Y. Xi, Increasing deaths from opioid analgesics in the United States, Pharmacoepidemiol Drug Saf, 15(9), 2006, 618-627.

[6] Department of Justice Drug Enforcement Administration, Automation of reports and consolidated orders system (ARCOS), http://www.deadiversion.usdoj.gov/arcos/index.html.

[7] Centers for Disease Control and Prevention, Community-based opioid overdose prevention programs providing naloxone-United States, 2010, MMWR, 61(6), 2012, 101-105.

[8] R.W. Hicks, J.P. Santell, D.D.Cousins, and R.L.Williams, MEDMARX 5th anniversary data report: a chartbook of 2003 findings and trends 1999-2003 (Rockville, MD: USP Center for the Advancement of Patient Safety, 2004).

[9] S.M. Dy, A.D. Shore, R.W. Hicks, and L.L. Morlock, Medication errors with opioids: results from a national reporting system, J Opioid Manag, 3(4), 2007, 189-194.

[10] E.W. Boyer, Management of opioid analgesic overdose, NEJM, 367(2), 2012, 146-155.

[11] H.D. Kleber, R.D. Weiss, R.F. Jr. Anton, T.P. George, S.F. Greenfield, T.R. Kosten, et al, Treatment of patients with substance use disorders, second edition, Am J Psychiatry, 163(8 Suppl), 2006, 5-82. 
[12] Part 10.2: Toxicolocy in ECC, American Heart Association, Circulation, 112, 2005, IV-126-IV-132.

[13] W.R. Martin, Naloxone, Ann Intern Med, 85, 1976, 765-768.

[14] S.F.J. Clarke, P.I. Dargan, and A.L. Jones, Naloxone in opioid poisoning: walking the tightrope, Emerg Med J, 22, 2005, 612-616.

[15] R.S. Hoffmann, and L.R. Goldfrank, The poisoned patient with altered consciousness: controversies in the use of a 'coma cocktail', JAMA, 274(7), 1995, 562-569.

[16] Naloxone package insert (Lake Forest, IL: Hospira, Inc., 2007).

[17] G.K. McEvoy (Ed.), Naloxone, AHFS drug information (Bethesda, MD: American Society of Health-System Pharmacists, 2004), 2093-2095.

[18] K.A. Sporer, J. Firestone, and S.M. Isaacs, Out-of-hospital treatment of opioid overdoses in an urban setting, Acad Emerg Med, 3(7), 1996, 660-667.

[19] K. Wanger, L. Brough, I. Macmillan, J. Goulding, I. MacPhail, and J.M. Christenson, Intravenous vs. subcutaneous naloxone for out-of hospital management of presumed opioid overdose, Acad Emerg Med, 5(4), 1998, 293-299.

[20] D. Kerr, A.M. Kelly, P. Dietze, D. Jolley, and B. Barger, Randomized controlled trial comparing the effectiveness and safety of intranasal and intramuscular naloxone for the treatment of suspected heroin overdose, Addiction, 104, 2009, 2067-2074.

[21] J.M. Weber, K.L. Tataris, J.D. Hoffman, S.E. Aks, and M.B. Mycyk, Can nebulized naloxone be used safely and effectively by emergency medical services for suspected opioid overdose? Prehosp Emerg Care, 16(2), 2012, 289292.

[22] M.D. Rios, M. Lanigan, and V. Zayas, Drugs of abuse: providing the best in evidence-based care to "selfmedicated" patients, in L. Freeman, A. Jagoda (Eds.), Emergency Medicine Practice, 7(5), 2005, 1-24.

[23] M.D. Saybolt, S.M. Alter, F. Dos Santos, D.P. Calello, K.O Rynn, D.A. Nelson, et al, Naloxone in cardiac arrest with suspected opioid overdoses, Resuscitation, 81(1), 2010, 42-46.

[24] W.A. Watson, M.T. Steele, R.L. Muelleman, and M.D. Rush, Opioid toxicity recurrence after an initial response to naloxone, J Toxicol Clin Toxicol, 36, 1998, 11-17.

[25] J, Christenson, J. Etherington, E. Grafstein, G. Innes, S. Pennington, K. Wanger, et al, Early discharge of patients with presumed opioid overdose: development of a clinical prediction rule, Acad Emerg Med, 7(10) 2000, 11101118 .

[26] J.J. Boyd, M.J. Kuisma, A.O. Alaspää, E. Vuori, J.V. Repo, and T.T. Randell, Recurrent opioid toxicity after prehospital care of presumed heroin overdose patients, Acta Anaesthesiol Scand, 50(10), 2006, 1266-1270.

[27] L. Goldfrank, R.S. Weisman, J.K Errick, and M.W. Lo, A dosing nomogram for continuous infusion intravenous naloxone, Ann Emerg Med, 15, 1986, 566-570.

[28] V. Waldron, C.R. Klimt, and J.E. Seibel, Methadone overdose treated with naloxone infusion, JAMA, 225(1), 1973, 53. 